\title{
Economic Dispatch Using Parameter-Setting-Free Harmony Search
}

\author{
Zong Woo Geem \\ Department of Energy and Information Technology, Gachon University, Seongnam 461-701, Republic of Korea \\ Correspondence should be addressed to Zong Woo Geem; geem@gachon.ac.kr
}

Received 5 February 2013; Revised 26 March 2013; Accepted 8 April 2013

Academic Editor: Xin-She Yang

Copyright (C) 2013 Zong Woo Geem. This is an open access article distributed under the Creative Commons Attribution License, which permits unrestricted use, distribution, and reproduction in any medium, provided the original work is properly cited.

\begin{abstract}
Economic dispatch is one of the popular energy system optimization problems. Recently, it has been solved by various phenomenon-mimicking metaheuristic algorithms such as genetic algorithm, tabu search, evolutionary programming, particle swarm optimization, harmony search, honey bee mating optimization, and firefly algorithm. However, those phenomenonmimicking problems require a tedious and troublesome process of algorithm parameter value setting. Without a proper parameter setting, good results cannot be guaranteed. Thus, this study adopts a newly developed parameter-setting-free technique combined with the harmony search algorithm and applies it to the economic dispatch problem for the first time, obtaining good results. Hopefully more researchers in energy system fields will adopt this user-friendly technique in their own problems in the future.
\end{abstract}

\section{Introduction}

Economic dispatch (ED) is defined in the US Energy Policy Act of 2005 as the operation of electrical generation facilities to produce energy at the least cost to reliably serve consumers while satisfying any operational limits of generation and transmission facilities. ED became a popular optimization problem in energy system field, which has been tackled by various optimization techniques such as genetic algorithm (GA) [1], tabu search (TS) [2], evolutionary programming (EP) [3], particle swarm optimization (PSO) [4], harmony search (HS) [5], honey bee mating optimization (HBMO) [6], and firefly algorithm (FA) [7].

As observed in the literature, better results have been obtained by phenomenon-mimicking metaheuristic algorithms rather than gradient-based mathematical techniques. Indeed, the metaheuristic algorithm has advantages over the mathematical technique in terms of several factors: (1) the former does not require complex derivative functions; (2) the former does not require a feasible starting solution vector which is sensitive to the final solution quality; and (3) the former has more chance to find the global optimum.
However, the metaheuristic algorithm also has the weakness in the sense that it requires "proper and appropriate" value setting for algorithm parameters [8]. For example, in GA, only carefully chosen values for crossover and mutation rates can guarantee good final solution quality, which is not an easy task for algorithm users in practical fields who seldom know how the algorithm exactly works.

In order to overcome this troublesome parameter setting process, researchers have proposed adaptive GA techniques [9], which adjust crossover and mutation rates adaptively, instead of using fixed rates, to find good solutions without manually setting the algorithm parameters. This adaptive technique has been applied to various technical applications such as environmental treatment [10], structural design [11], and sewer network design [12].

In energy system field, the adaptive GA was also applied to a reactive power dispatch optimization as early as 1998 [13]. Afterwards, however, there have been seldom applications in major research databases using the adaptive technique. Thus, this study intends to apply a newly developed adaptive parameter-setting-free (PSF) technique [8], which is combined with the HS algorithm, to the economic dispatch problem for the first time. 


\section{Economic Dispatch Problem}

The economic dispatch problem can be optimally formulated. The objective function can be as follows:

$$
\operatorname{Min} z=\sum_{i} C_{i}\left(P_{i}\right)
$$

where $C_{i}(\cdot)$ is generation cost for generator $i$ and $P_{i}$ is electrical power generated by generator $i$. Here, $C_{i}(\cdot)$ can be further expressed as follows:

$$
C_{i}\left(P_{i}\right)=a_{i}+b_{i} P_{i}+c_{i} P_{i}^{2}+\left|e_{i} \times \sin \left(f_{i} \times\left(P_{i}^{\min }-P_{i}\right)\right)\right|,
$$

where $a_{i}, b_{i}, c_{i}, e_{i}$, and $f_{i}$ are cost coefficients for generator $i$. The fourth term in the right-hand side of (2) represents valvepoint effects.

The above objective function is to be minimized while satisfying the following equality constraint:

$$
\sum_{i} P_{i}=D
$$

where $D$ is total load demand. Also, each generator should generate power between minimum and maximum limits as the following inequality constraint:

$$
P_{i}^{\min } \leq P_{i} \leq P_{i}^{\max } .
$$

\section{Parameter-Setting-Free Technique}

The parameter-setting-free harmony search (PSF-HS) algorithm was first proposed for optimizing the discrete-variable problems such as structural design [14], water network design [15], and recreational magic square [8]. PSF-HS was also applied to a continuous-variable problem such as hydrologic parameter calibration [16].

However, it was never applied to a continuous-variable problem with technical constraints. Thus, this study first applies PSF-HS to the ED problem, whose type is the continuous-variable problem with a technical constraint, because its decision variable $P_{i}$ has the continuous value and it has the equality constraint of total power demand as expressed in (3). Here, the inequality constraint in (4) can be simply considered as value ranges without using any penalty method.

The basic HS algorithm manages a memory matrix, named harmony memory, as follows:

$$
\mathbf{H M}=\left[\begin{array}{cccc|c}
P_{1}^{1} & P_{2}^{1} & \cdots & P_{n}^{1} & z\left(\mathbf{P}^{1}\right) \\
P_{1}^{2} & P_{2}^{2} & \cdots & P_{n}^{2} & z\left(\mathbf{P}^{2}\right) \\
\vdots & \cdots & \cdots & \cdots & \vdots \\
P_{1}^{\mathrm{HMS}} & P_{2}^{\mathrm{HMS}} & \cdots & P_{n}^{\mathrm{HMS}} & z\left(\mathbf{P}^{\mathrm{HMS}}\right)
\end{array}\right] .
$$

Once this $\mathbf{H M}$ is fully filled with randomly generated vectors $\left(\mathbf{P}^{1}, \ldots, \mathbf{P}^{\mathrm{HMS}}\right)$, a new vector $\mathbf{P}^{\mathrm{New}}$ is generated as follows:

$$
P_{i}^{\mathrm{New}} \longleftarrow \begin{cases}P_{i}^{\min } \leq P_{i} \leq P_{i}^{\max } & \text { w.p. } R_{\text {Random }} \\ P_{i}(k) \in\left\{P_{i}^{1}, P_{i}^{2}, \ldots, P_{i}^{\mathrm{HMS}}\right\} & \text { w.p. } R_{\text {Memory }} \\ P_{i}(k)+\Delta & \text { w.p. } R_{\text {Pitch }}\end{cases}
$$

where $R_{\text {Random }}$ is random selection rate, $R_{\text {Memory }}$ is pure memory consideration rate, $R_{\text {Pitch }}$ is pure pitch adjustment rate, and $\Delta$ is pitch adjustment amount.

If the newly generated vector $\mathbf{P}^{\mathrm{New}}$ is better than the worst vector $\mathbf{P}^{\text {Worst }}$ in $\mathbf{H M}$, those two vectors are swapped as follows:

$$
\mathbf{P}^{\text {New }} \in \mathbf{H M} \wedge \mathbf{P}^{\text {Worst }} \notin \mathbf{H M} \text {. }
$$

The basic HS algorithm performs (6) and (7) until a termination criterion is satisfied.

For PSF-HS, one additional matrix, named operation type matrix (OTM), is also managed as follows:

$$
\left[\begin{array}{cccc}
o_{1}^{1}=\text { Random } & o_{2}^{1}=\text { Pitch } & \cdots & o_{n}^{1}=\text { Memory } \\
o_{1}^{2}=\text { Memory } & o_{2}^{2}=\text { Memory } & \cdots & o_{n}^{2}=\text { Pitch } \\
\vdots & \cdots & \cdots & \cdots \\
o_{1}^{\mathrm{HMS}}=\text { Memory } & o_{2}^{\mathrm{HMS}}=\text { Random } & \cdots & o_{n}^{\mathrm{HMS}}=\text { Memory }
\end{array}\right]
$$

OTM memorizes which operation (random selection, memory consideration, and pitch adjustment) each value comes from. For example, if the value of $P_{2}^{2}$ in $\mathbf{H M}$ comes from memory consideration operation, the value of $o_{2}^{2}$ in OTM is also set as "Memory." This process happens when initial vectors are populated or when a new vector is inserted into HM.

Thus, instead of using fixed algorithm parameter values, PSF-HS can utilize adaptive parameter values by calculating them at each iteration as follows:

$$
\begin{gathered}
R_{i, \text { Random }}=\frac{c t\left(o_{i}^{j}=\text { Random }, j=1,2, \ldots, \mathrm{HMS}\right)}{\mathrm{HMS}}, \\
R_{i, \text { Memory }}=\frac{c t\left(o_{i}^{j}=\text { Memory, } j=1,2, \ldots, \mathrm{HMS}\right)}{\mathrm{HMS}}, \quad i=1,2, \ldots, n, \\
R_{i, \text { Pitch }}=\frac{c t\left(o_{i}^{j}=\text { Pitch, } j=1,2, \ldots, \mathrm{HMS}\right)}{\mathrm{HMS}}, \quad i=1,2, \ldots, n,
\end{gathered}
$$

where $c t(\cdot)$ is a function which counts specific elements that satisfy the condition.

\section{Numerical Example}

The PSF-HS is applied to a popular bench-mark ED problem with three generators. The input data for the three-generator problem is shown in Table 1.

When the total system demand is set to $850 \mathrm{MW}$, the optimal solution is known as $\$ 8234.07$ [2-4], which was replicated by using a popular gradient-based technique (generalized reduced gradient (GRG) method), which has 
TABLE 1: Data for three-generator example with valve-point loading.

\begin{tabular}{lccccccc}
\hline Generator & $P_{i}^{\min }$ & $P_{i}^{\max }$ & $a_{i}$ & $b_{i}$ & $c_{i}$ & $e_{i}$ & $f_{i}$ \\
\hline 1 & 100 & 600 & 0.001562 & 7.92 & 561 & 300 & 0.0315 \\
2 & 50 & 200 & 0.00482 & 7.97 & 78 & 150 & 0.063 \\
3 & 100 & 400 & 0.00194 & 7.85 & 310 & 200 & 0.042 \\
\hline
\end{tabular}

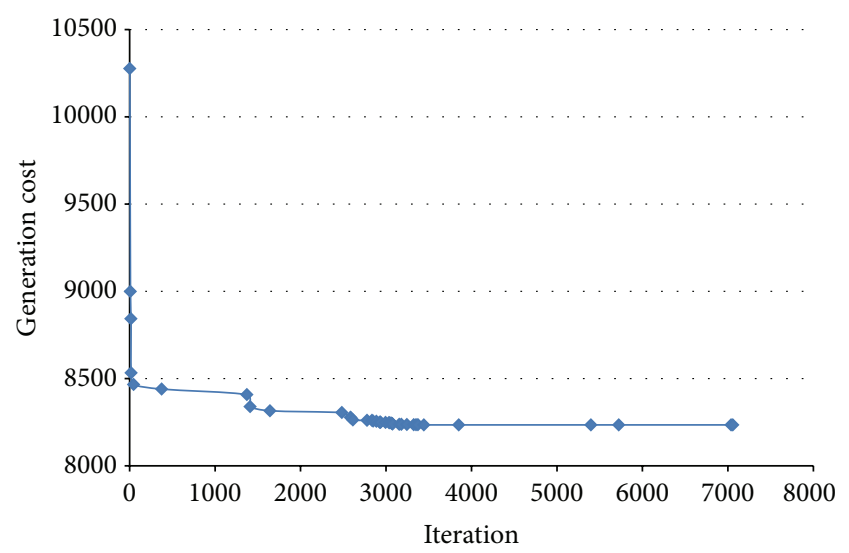

FIgURE 1: Convergence History of Generation Cost.

been also successfully applied to other energy optimization problems such as building chiller loading [17], combined heat and power ED [18], and hybrid renewable energy system design [19]. However, the GRG method was able to obtain the identical best solution only when it started with a vector $\left(P_{1}=300 ; P_{2}=150 ; P_{3}=400\right)$. Instead, when, different starting vector $\left(P_{1}=600, P_{2}=200, P_{3}=400\right)$ was used, solution quality was worsened as $\$ 8241.41$.

When PSF-HS was also applied to the problem, it obtained a near-optimal solution of $\$ 8234.47$ after 100 runs, which has small discrepancy from the optimal solution ( $\$ 8234.07$ ) by $0.005 \%$. For the results from 100 runs, maximum and mean solutions are $\$ 8429.74$ (2.4\% discrepancy) and $\$ 8292.88$ (0.7\% discrepancy), respectively. Here, PSFHS was performed using MS-Excel VBA environment with Intel CPU 3.3 GHz. Each run takes only one second in this computing environment.

Figure 1 shows the convergence history of power generation cost for the case of the near-optimal solution $\$ 8234.47$. As seen in the figure, PSF-HS closely approached to the nearoptimal solution in early iterations.

Table 2 shows the final HM with HMS $=30$. As observed in the table, there are many similar vectors in HM because PSF-HS tried local search, instead of global search, in late stage of computation.

Figure 2 shows the history of random selection rate $R_{\text {Random }}$. As observed in the figure, all three parameters ( $R_{1, \text { Random }}, R_{2, \text { Random }}$, and $R_{3, \text { Random }}$ ) started with higher values (0.5). In less than 1,000 iterations, $R_{1, \text { Random }}$ went up to around $0.4, R_{2, \text { Random }}$ to around 0.5 , and $R_{3, \text { Random }}$ to around 0.8 . Then, they abruptly wend down to less than 0.1 after 3,000 iterations.

Figure 3 shows the history of pure memory consideration rate $R_{\text {Memory }}$. As observed in the figure, all three parameters
TABLE 2: Values of final HM.

\begin{tabular}{lccccc}
\hline Number & $P_{1}$ & $P_{2}$ & $P_{3}$ & $\sum_{i} P_{i}$ & $\sum_{i} C_{i}\left(P_{i}\right)$ \\
\hline 1 & 300.944 & 149.782 & 399.274 & 850.000 & 8234.472 \\
2 & 300.944 & 149.782 & 399.274 & 850.001 & 8234.477 \\
3 & 300.944 & 149.782 & 399.274 & 850.001 & 8234.479 \\
4 & 300.973 & 149.754 & 399.274 & 850.002 & 8234.481 \\
5 & 301.006 & 149.751 & 399.244 & 850.001 & 8234.482 \\
6 & 300.974 & 149.782 & 399.244 & 850.000 & 8234.483 \\
7 & 300.974 & 149.754 & 399.274 & 850.002 & 8234.487 \\
8 & 300.977 & 149.779 & 399.244 & 850.001 & 8234.489 \\
9 & 300.977 & 149.751 & 399.274 & 850.003 & 8234.496 \\
10 & 300.945 & 149.782 & 399.274 & 850.002 & 8234.497 \\
11 & 300.912 & 149.815 & 399.274 & 850.001 & 8234.501 \\
12 & 300.934 & 149.822 & 399.244 & 850.000 & 8234.509 \\
13 & 300.973 & 149.784 & 399.244 & 850.002 & 8234.510 \\
14 & 300.944 & 149.784 & 399.274 & 850.002 & 8234.511 \\
15 & 300.912 & 149.815 & 399.274 & 850.002 & 8234.511 \\
16 & 300.934 & 149.794 & 399.274 & 850.002 & 8234.511 \\
17 & 300.905 & 149.822 & 399.274 & 850.001 & 8234.514 \\
18 & 300.974 & 149.784 & 399.244 & 850.002 & 8234.516 \\
19 & 300.944 & 149.784 & 399.274 & 850.003 & 8234.517 \\
20 & 301.013 & 149.786 & 399.202 & 850.001 & 8234.520 \\
21 & 301.013 & 149.786 & 399.202 & 850.002 & 8234.535 \\
22 & 300.945 & 149.784 & 399.274 & 850.004 & 8234.535 \\
23 & 301.009 & 149.751 & 399.244 & 850.004 & 8234.536 \\
24 & 301.006 & 149.754 & 399.244 & 850.004 & 8234.538 \\
25 & 300.973 & 149.786 & 399.244 & 850.003 & 8234.542 \\
26 & 300.977 & 149.782 & 399.244 & 850.003 & 8234.542 \\
27 & 300.944 & 149.786 & 399.274 & 850.004 & 8234.542 \\
28 & 301.006 & 149.794 & 399.202 & 850.002 & 8234.543 \\
29 & 300.977 & 149.782 & 399.244 & 850.004 & 8234.547 \\
30 & 300.974 & 149.786 & 399.244 & 850.004 & 8234.548 \\
\hline & & & & &
\end{tabular}

$\left(R_{1, \text { Memory }}, R_{2, \text { Memory }}\right.$ and $\left.R_{3, \text { Memory }}\right)$ abruptly went up from the starting point of 0.25 . After 4,000 iterations, they became more than 0.8 and stayed.

Figure 4 shows the history of pure pitch adjustment rate $R_{\text {Pitch }}$. As observed in the figure, all three parameters $\left(R_{1, \text { Pitch }}, R_{2, \text { Pitch }}\right.$, and $\left.R_{3, \text { Pitch }}\right)$, from the starting point of 0.25 , monotonically stayed less than 0.3 except for one situation when $R_{3 \text {,Pitch }}$ spiked near 3,000 iterations.

Furthermore, the sensitivity analysis of initial parameter values was performed. While the original parameter set $\left(R_{\text {Random }}=0.5, R_{\text {Memory }}=0.25\right.$, and $\left.R_{\text {Pitch }}=0.25\right)$ resulted in minimal solution of $\$ 8,243.56$ and average solution of $\$ 8,287.69$ after 10 runs, equal-valued parameter set $\left(R_{\text {Random }}=0.33, R_{\text {Memory }}=0.33\right.$, and $\left.R_{\text {Pitch }}=0.33\right)$ resulted in minimal solution of $\$ 8,242.12$ and average solution of $\$ 8,322.11$; memory-consideration-oriented parameter set $\left(R_{\text {Random }}=0.1, R_{\text {Memory }}=0.7\right.$, and $\left.R_{\text {Pitch }}=0.2\right)$ resulted in minimal solution of $\$ 8,241.34$ and average solution of $\$ 8,314.45$; random-selection-oriented parameter set $\left(R_{\text {Random }}=0.8, R_{\text {Memory }}=0.1\right.$, and $\left.R_{\text {Pitch }}=0.1\right)$ resulted in minimal solution of $\$ 8,241.29$ and average solution of 


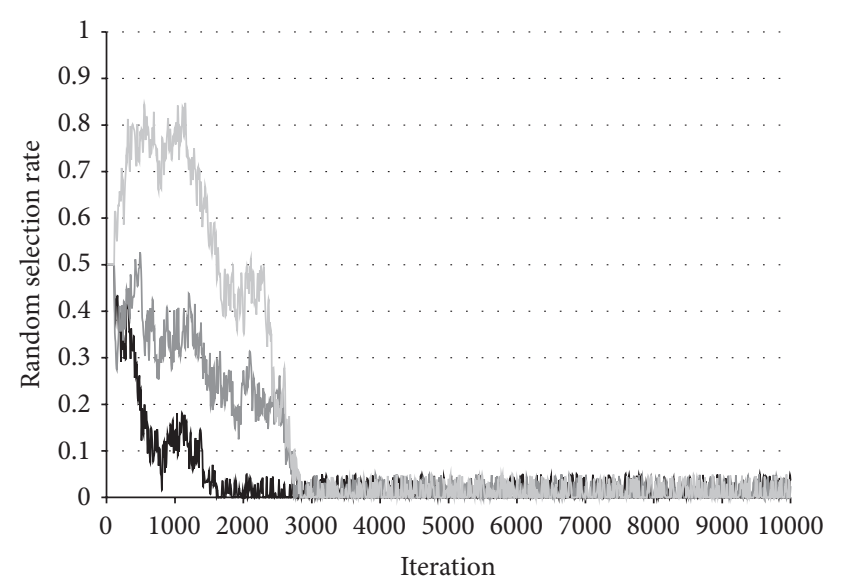

$-P_{1}$
$-P_{2}$
$-P_{3}$

FIgURE 2: History of Random Selection Rate.

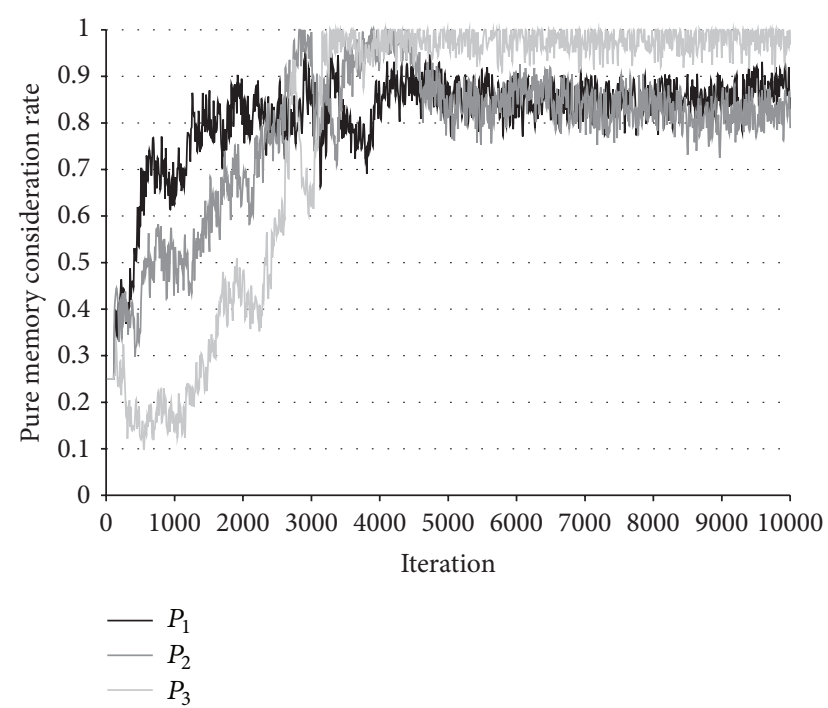

Figure 3: History of Pure Memory Consideration Rate.

$\$ 8,272.40$. It appeared that the initial parameter values are not very sensitive to final solution quality.

Especially, when the results from memory-considerationoriented parameter set $\left(R_{\text {Random }}=0.1, R_{\text {Memory }}=0.7\right.$, and $R_{\text {Pitch }}=0.2$ ) and those from random-selection-oriented parameter set $\left(R_{\text {Random }}=0.8, R_{\text {Memory }}=0.1\right.$, and $R_{\text {Pitch }}=$ $0.1)$ were statistically compared, although their variances are different based on $F$-test $(p=0.04)$, their averages are not significantly different based on $t$-test $(p=0.16)$.

\section{Conclusions}

This study applied PSF-HS to the ED problem for the first time, obtaining a good solution which is very close to the best solution ever found. While existing metaheuristic algorithms require carefully chosen algorithm parameters,

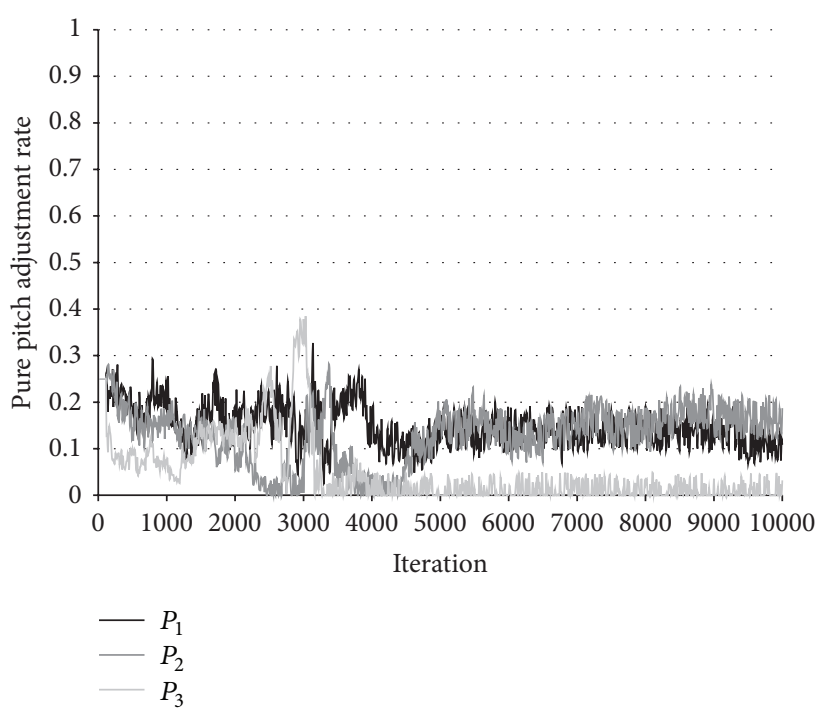

FIgure 4: History of Pure Pitch Adjustment Rate.

PSF-HS did not require that tedious process. Thus, there surely exists a tradeoff between original HS and PSF-HS. Also, it should be noted that PSF-HS respectively considers individual algorithm parameters for each variable, which is more efficient way than using lumped parameters for all variables.

For future study, the structure of PSF-HS should be improved to do better performance. Also, it can be applied to large-scale real-world problems to test scalability. Also, other researchers are expected to apply this novel technique to their own energy-related problems.

\section{Acknowledgment}

This work was supported by the Gachon University Research Fund of 2013 (GCU-2013-R114).

\section{References}

[1] D. C. Walters and G. B. Sheble, "Genetic algorithm solution of economic dispatch with value point loading," IEEE Transactions on Power Systems, vol. 8, no. 3, pp. 1325-1332, 1993.

[2] W.-M. Lin, F.-S. Cheng, and M.-T. Tsay, "An improved tabu search for economic dispatch with multiple minima," IEEE Transactions on Power Systems, vol. 17, no. 1, pp. 108-112, 2002.

[3] N. Sinha, R. Chakrabarti, and P. K. Chattopadhyay, "Evolutionary programming techniques for economic load dispatch," IEEE Transactions on Evolutionary Computation, vol. 7, no. 1, pp. 8394, 2003.

[4] J.-B. Park, K.-S. Lee, J.-R. Shin, and K. Y. Lee, "A particle swarm optimization for economic dispatch with nonsmooth cost functions," IEEE Transactions on Power Systems, vol. 20, no. 1, pp. 34-42, 2005.

[5] B. K. Panigrahi, V. R. Pandi, S. Das, Z. Cui, and R. Sharma, "Economic load dispatch using population-variance harmony search algorithm," Transactions of the Institute of Measurement and Control, vol. 34, no. 6, pp. 746-754, 2012. 
[6] T. Niknam, H. D. Mojarrad, H. Z. Meymand, and B. B. Firouzi, "A new honey bee mating optimization algorithm for nonsmooth economic dispatch," Energy, vol. 36, no. 2, pp. 896-908, 2011.

[7] X.-S. Yang, S. S. S. Hosseini, and A. H. Gandomi, "Firefly Algorithm for solving non-convex economic dispatch problems with valve loading effect," Applied Soft Computing, vol. 12, no. 3, pp. 1180-1186, 2012.

[8] Z. W. Geem and K.-B. Sim, "Parameter-setting-free harmony search algorithm," Applied Mathematics and Computation, vol. 217, no. 8, pp. 3881-3889, 2010.

[9] M. Srinivas and L. M. Patnaik, "Adaptive probabilities of crossover and mutation in genetic algorithms," IEEE Transactions on Systems, Man and Cybernetics, vol. 24, no. 4, pp. 656667, 1994.

[10] M. S. Gibbs, H. R. Maier, and G. C. Dandy, "Comparison of genetic algorithm parameter setting methods for chlorine injection optimization," Journal of Water Resources Planning and Management, vol. 136, no. 2, pp. 288-291, 2010.

[11] S. Bekiroğlu, T. Dede, and Y. Ayvaz, "Implementation of different encoding types on structural optimization based on adaptive genetic algorithm," Finite Elements in Analysis and Design, vol. 45, no. 11, pp. 826-835, 2009.

[12] A. Haghighi and A. E. Bakhshipour, "Optimization of sewer networks using an adaptive genetic algorithm," Water Resources Management, vol. 26, no. 12, pp. 3441-3456, 2012.

[13] Q. H. Wu, Y. J. Cao, and J. Y. Wen, "Optimal reactive power dispatch using an adaptive genetic algorithm," International Journal of Electrical Power and Energy Systems, vol. 20, no. 8, pp. 563-569, 1998.

[14] O. Hasanebi, F. Erdal, and M. P. Saka, "Adaptive harmony search method for structural optimization," Journal of Structural Engineering, vol. 136, no. 4, pp. 419-431, 2010.

[15] Z. W. Geem and Y. H. Cho, "Optimal design of water distribution networks using parameter-setting-free harmony search for two major parameters," Journal of Water Resources Planning and Management, vol. 137, no. 4, pp. 377-380, 2011.

[16] Z. W. Geem, "Parameter estimation of the nonlinear muskingum model using parameter-setting-free harmony search," Journal of Hydrologic Engineering, vol. 16, no. 8, pp. 684-688, 2011.

[17] Z. W. Geem, "Solution quality improvement in chiller loading optimization," Applied Thermal Engineering, vol. 31, no. 10, pp. 1848-1851, 2011.

[18] Z. W. Geem and Y. H. Cho, "Handling non-convex heat-power feasible region in combined heat and power economic dispatch," International Journal of Electrical Power \& Energy Systems, vol. 34, no. 1, pp. 171-173, 2012.

[19] Z. W. Geem, "Size optimization for a hybrid photovoltaicwind energy system," International Journal of Electrical Power \& Energy Systems, vol. 42, no. 1, pp. 448-451, 2012. 


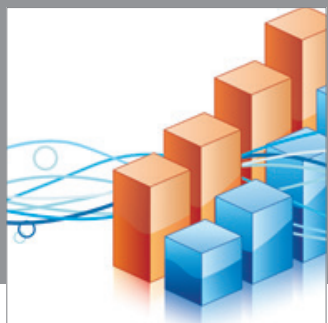

Advances in

Operations Research

mansans

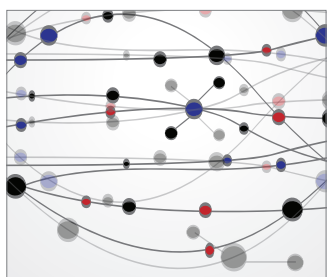

The Scientific World Journal
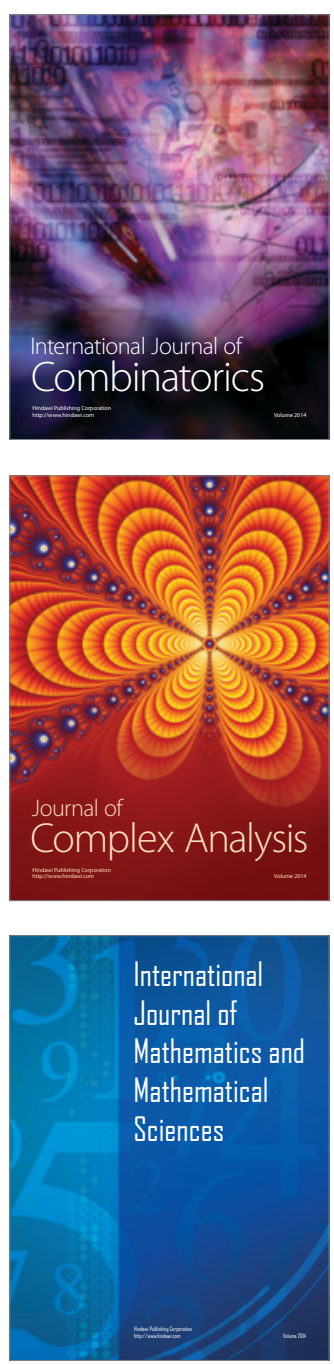
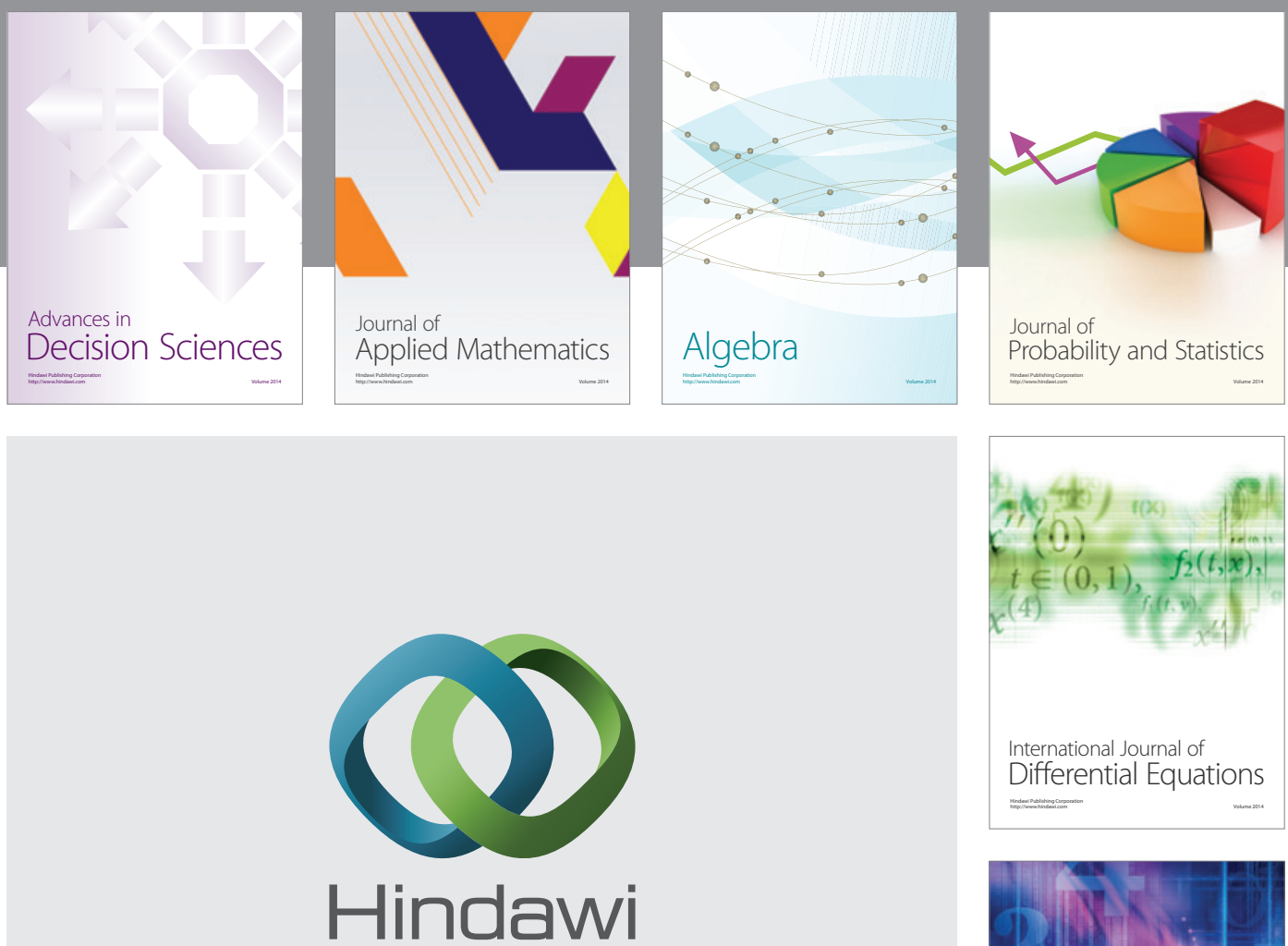

Submit your manuscripts at http://www.hindawi.com
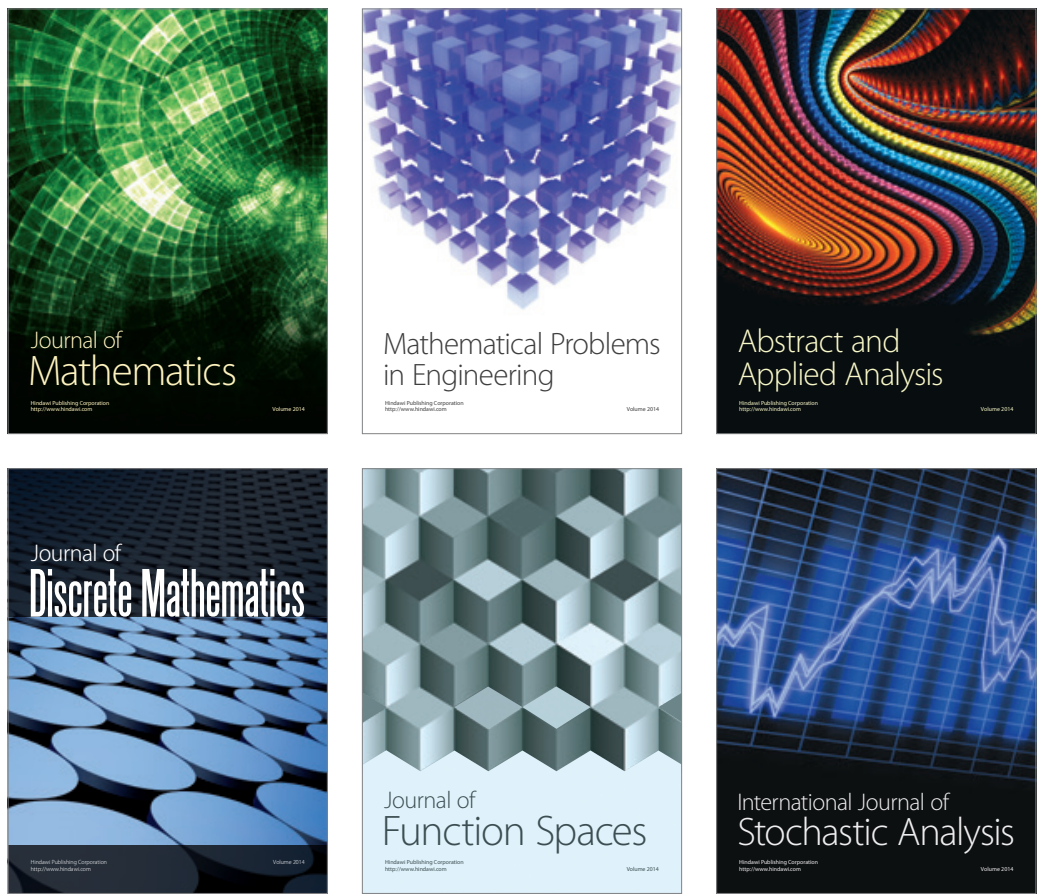

Journal of

Function Spaces

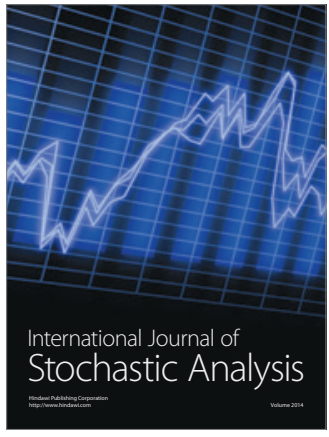

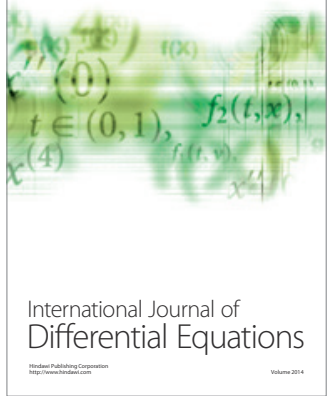
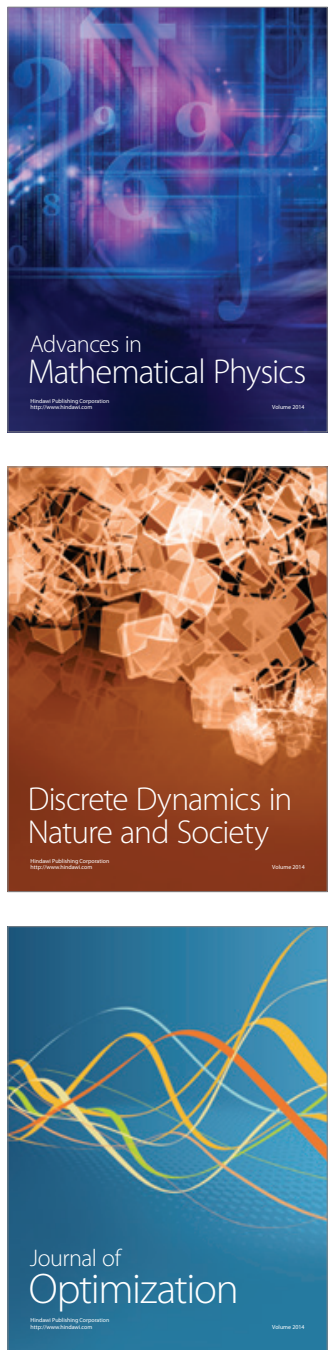